\title{
CONHECIMENTO DOS PROFISSIONAIS DE ENFERMAGEM NO MANEJO DA DOR DE PACIENTES ONCOLÓGICOS
}

\author{
KNOWLEDGE OF PROFESSIONAL NURSING IN THE MANAGEMENT OF PAIN CANCER \\ PATIENTS
}

\author{
Hellen Cristina Almeida Abreu de Lara ${ }^{a^{*}}$, Elizete Aparecida Rubira ${ }^{b^{* *}}$, \\ Samira Reschetti Marcon ${ }^{\mathrm{c}^{* *}}$, José Roberto Temponi de Oliveira ${ }^{\mathrm{d}^{* \star}}$, Alyne Silva Britto ${ }^{\mathrm{e}^{* *}}$, \\ Roselma Marcele da Silva Alexandre ${ }^{f^{*}}$ \\ afa_nany@yahoo.com.bra ${ }^{\text {a }}$, earubira@gmail.com ${ }^{\mathrm{b}}$, samira.marcon@gmail.com ${ }^{\mathrm{c}}$, temponi30@gmail.com ${ }^{\mathrm{d}}$, \\ alyne.britto@gmail.com ${ }^{\mathrm{e}}$, roselma_marcele@hotmail.com ${ }^{\mathrm{f}}$ \\ Centro Universitário de Várzea Grande (UNIVAG)*, Universidade Federal de Mato Grosso**
}

Data do recebimento do artigo: 11/07/2018

Data do aceite: $08 / 10 / 2018$

\begin{abstract}
RESUMO
Introdução: $\mathrm{O}$ controle da dor é um dos desafios para o câncer, dentre os profissionais envolvidos nesse manuseio é a equipe de enfermagem. Dessa forma, nota-se a necessidade de avaliações do conhecimento de enfermagem em relação à dor dos pacientes em tratamento oncológico.Objetivo: identificar o conhecimento dos profissionais de enfermagem no manejo da dor de pacientes portadores de neoplasias malignas em tratamento clínico e cirúrgico. Método: Estudo transversal, realizado com os profissionais de enfermagem das clínicas médico e cirúrgica. Foram utilizados 2 questionários: $1^{\circ}$ composto por questões abertas e subjetivas, e o $2^{\circ}$ questões fechadas e objetivas. Resultados: Entre os profissionais, 60 \% conheciam os tipos de dor. Quanto ao conhecimento sobre as escalas existentes, no questionário 1, relataram que conhece as escalas de dor existentes, e 63,6\% disseram que não conhecem. No entanto, no questionário $236,1 \%$ relataram que não conhecem. $\mathrm{O}$ grau de concordância entre os questionários, tornou claro a falta de conhecimento no manejo da dor em ambos questionários, pois tanto as respostas subjetivas como as objetivas mostraram baixo conhecimento no manejo e tratamento da dor. Conclusão: Evidenciou que a equipe de enfermagem deve ser treinada no que se refere ao manejo da dor, para que seja possível atrelar conhecimento e ação.
\end{abstract}

Palavras chave: Enfermagem; neoplasias; dor; equipe de enfermagem

\begin{abstract}
Introduction: Pain control is one of the challenges for cancer, among the professionals involved in this handling is the nursing team. Thus, we note the need for assessments of nursing knowledge regarding the pain of patients on cancer treatment. Objective: To identify the knowledge of nurses in pain management of patients with malignancies in clinical and surgical treatment. Method: Cross-sectional study conducted among nursing professionals of medical and surgical clinics. 1 consisting of open and subjective questions, and the 2nd closed and objective questions: two questionnaires were used. Results: Among the professionals, $60 \%$ knew the types of pain. Regarding knowledge of existing scales, the questionnaire first reported that knows the scales of pain, and $63.6 \%$ said they did not know. However, the second questionnaire reported that $36.1 \%$ do not know. The degree of agreement between the questionnaires, made clear the lack of knowledge about pain management in both questionnaires, as both subjective and objective responses showed low knowledge in the management and treatment of pain. Conclusion: There was that nursing staff should be trained with regard to pain management, so you can harness knowledge and action.
\end{abstract}

Keywords: Pain; neoplasms; nursing; nursing team 


\section{INTRODUÇÃO}

O câncer é uma doença que causa desconforto e sofrimento aqueles que o vivenciam, decorrente de diversos fatores, dentre eles: presença do tumor, desgaste físico, emocional, espiritual ou social advindos do tratamento ou pelos sintomas impostos pela doença. Dentre os desconfortos experimentados pelos pacientes com câncer, a dor é apontada como muito comum, acometendo cerca de $50 \%$ dos pacientes em todos os estágios da doença, $10 \%$ a $15 \%$ com intensidade significativa já no estágio inicial e com o aparecimento de metástase em torno de $70 \%{ }^{1,2}$.

A dor acarreta grande estresse e sofrimento aos doentes e aos que os rodeiam. Este quadro, repetidamente, resulta em perspectivas emocionais, sociais e econômicas desfavoráveis ao doente e sua família. Em alguns casos, a dor do câncer não é gerenciada, ocorrendo impacto negativo na sobrevida do paciente, que manifesta maior medo da dor, do sofrimento, do que propriamente de morrer. A família e os amigos também sofrem, pois assistem a dor e a angústia vivida por um ente querido ${ }^{2,3}$.

A relação entre a dor e outras causas de sofrimento tem importantes implicações na prática clínica e o seu tratamento deve fazer parte de uma abordagem multidisciplinar eficaz. Para isso, é imprescindível o conhecimento dos mecanismos fisiopatológicos, aspectos psicossociais e emocionais relacionados à doença, métodos utilizados para sua avaliação e terapêuticas disponíveis para seu controle ${ }^{1-3}$.

Pesquisas mostram que os profissionais da saúde têm dificuldade em identificar e tratar a dor. A dificuldade pode estar relacionada à condição do paciente oncológico impossibilitado ou com dificuldades para referir e/ou comunicar seu sofrimento por restrições impostas pelos métodos terapêuticos, e a falta de conhecimento científico, também pode ser considerada uma razão para o manuseio e controle inadequado da dor ${ }^{4}$.

O conhecimento dos profissionais de enfermagem sobre a dor constitui em um dos principais fatores para a promoção de conforto e alívio da dor, visto que esses profissionais são os que permanecem a maior parte do tempo junto a esses pacientes. Durante a internação, a enfermagem é a equipe que mantém maior contato com o pa- ciente e familiares, participando das rotinas e procedimentos 24 horas por dia, vivenciando dores e sofrimento, buscando contribuir para o conforto e alivio dessas situações. Assim, a necessidade de prover alívio da dor, do estresse e do desconforto aos pacientes deve ser uma constante na vida na equipe de enfermagem ${ }^{2}$.

Assim, o objetivo deste estudo foi identificar o conhecimento dos profissionais de enfermagem no manejo da dor de pacientes portadores de neoplasias malignas em tratamento clínico e cirúrgico, em um serviço especializado no tratamento do câncer em Mato Grosso.

\section{MÉTODO}

Pesquisa quantitativa com delineamento transversal, realizada nas clínicas médica e cirúrgica, de um hospital especializado em tratamento de câncer, no Estado de Mato Grosso. A escolha dessas unidades se deu pelo fato das mesmas possuírem maior número de leito, onde os pacientes em algum momento irão vivenciar algum tipo de dor.

Quarenta e cinco profissionais de enfermagem estão lotados nestas unidades, e os critérios de inclusão foram: prestar assistência direta ao paciente e estar trabalhando no período da coleta de dados. Um enfermeiro, cinco técnicos de enfermagem se recusaram a participar da pesquisa e dois técnicos estavam afastados por licença médica no período da coleta. Assim a população foi composta por trinta e seis profissionais de enfermagem.

A coleta de dados foi realizada no período de 22 de janeiro a 01 de fevereiro de 2012, nos turnos matutino, vespertino e noturno. Foram utilizados dois questionários elaborados pelas pesquisadoras: o primeiro composto por questões abertas e subjetivas, e o segundo feito por questões fechadas e objetivas. Cada instrumento era composto exatamente das mesmas perguntas e constituído de duas partes: a primeira com os dados sociodemográficos e profissionais, e a segunda sobre o conhecimento do manejo da dor. Os questionários foram respondidos e preenchidos por cada sujeito em dois momentos distintos, evitando que as alternativas do questionário fechado (Q2), interferissem no preenchimento do questionário aberto (Q1). 
A pesquisa foi aprovada pelo Comitê de Ética e Pesquisa $n^{\circ} 137 / 2011$ e os sujeitos assinaram o Termo de Consentimento Livre e Esclarecido.

Os dados foram digitados e analisados no programa SPSS 17.0, realizado análises descritivas e inferenciais para verificar a correlação entre as variáveis. Para descrever a intensidade da concordância entre os dois questionários, utilizou-se uma adaptação da medida Kappa que é baseada no número de respostas concordantes.

\section{RESULTADOS}

Entre os sujeitos deste estudo, 83,3\% eram técnicos de enfermagem, 2,8\% auxiliar de enfermagem e $13,0 \%$ enfermeiros, $88,9 \%$ do sexo feminino, a faixa etária variou de 21 a 53 anos, com média de 34,2 anos. Nenhum profissional relatou ter feito pós-graduação (lato-senso) na área de oncologia, e a grande proporção $(77,8 \%)$, nunca havia realizado cursos de atualização na área, demais dados encontra-se na tabela 1 .

Tabela 1 - Perfil dos profissionais de enfermagem quanto ao tempo de exercício profissional e tempo de experiência cuidando de pacientes com câncer, Cuiabá-MT, 2012

\begin{tabular}{|c|c|c|c|c|c|c|c|c|c|c|c|c|}
\hline \multirow[t]{2}{*}{ Categoria } & \multicolumn{6}{|c|}{ Tempo de exercício profissional } & \multicolumn{6}{|c|}{$\begin{array}{l}\text { Tempo de experiência cuidando } \\
\text { de pacientes com câncer }\end{array}$} \\
\hline & $\mathrm{n}^{<}$ & $\begin{array}{l}\text { nos } \\
\%\end{array}$ & $\stackrel{\mathrm{En}}{\mathrm{N}}$ & a 5 & $\mathrm{~N}^{3}$ & $\begin{array}{l}105 \\
\%\end{array}$ & $\mathrm{~N}^{<}$ & $\begin{array}{l}10 \mathrm{~s} \\
\%\end{array}$ & $\stackrel{\mathrm{En}}{\mathrm{N}}$ & a 5 & $\mathrm{n}$ & $\begin{array}{c}105 \\
\%\end{array}$ \\
\hline $\begin{array}{l}\text { Auxiliar de enfer- } \\
\text { magem }\end{array}$ & - & - & - & - & 1 & 27,8 & - & - & - & - & 1 & 2,8 \\
\hline $\begin{array}{l}\text { Técnico (a) de } \\
\text { enfermagem }\end{array}$ & 7 & 19,4 & 13 & 36,1 & 10 & 27,8 & 11 & 30,6 & 12 & 33,3 & 7 & 19,4 \\
\hline Enfermeiro (a) & 3 & 8.3 & 1 & 2,8 & 1 & 2,8 & 5 & 13,9 & - & - & - & - \\
\hline Total & 10 & 27,8 & 14 & 38,9 & 12 & 33,3 & 16 & 44,5 & 12 & 33,3 & 8 & 22,2 \\
\hline
\end{tabular}

A Tabela 2 refere-se ao conhecimento dos tipos de dor nos dois instrumentos, evidencia que não existe diferença entre as respostas do Q1 e Q2 $(\mathrm{p}=0,559)$, pois $\mathrm{Q} 160 \%$ afirmaram conhecer os tipos de dor, e 40\% desconheciam. No Q2, 69,4\% relataram que conheciam os tipos de dor e 30,6\% desconheciam. A dor crônica e a dor aguda foram as mais citadas em ambos os questionários.

Quanto ao conhecimento sobre as escalas de dor existentes, houve uma inversão de respostas, demonstrando que a dependência entre o tipo de questionário ( $\mathrm{p}=0,012)$. No Q1 36,4\% conheciam as escalas, e 63,6\% não conheciam. No entanto, em Q2 63,9\% conheciam e 36,1\% não conheciam, demais dados na tabela abaixo.

Em relação à utilização de escalas de dor durante o cuidado, evidenciou que não existiu diferença entre as respostas do Q1 e Q2 $(\mathrm{p}=0,559)$. No Q1 50,0\% e Q2 86,9\% utilizavam as escalas de dor durante o cuidado.

Tabela 2- Conhecimento dos tipos dor e de escalas de avaliação relatado por profissionais de enfermagem, Cuiabá-MT, 2012

\begin{tabular}{|c|c|c|c|c|c|c|}
\hline \multicolumn{2}{|l|}{ Variáveis } & \multicolumn{2}{|c|}{ Q1 } & \multicolumn{2}{|r|}{ Q2 } & \multirow[t]{2}{*}{$\mathrm{p}$} \\
\hline & & $\mathbf{n}^{*}$ & $\%$ & $\mathbf{n}^{\star}$ & $\%$ & \\
\hline Conhecimento dos Tipos de & Sim & 21 & 60 & 25 & 69,4 & 0,559 \\
\hline dor & Não & 14 & 40 & 11 & 30,6 & \\
\hline $\begin{array}{l}\text { Tipos de dor mais citadas (den- } \\
\text { tre os que responderam sim) }\end{array}$ & $\begin{array}{l}\text { Dor Crônica } \\
\text { Dor Aguda } \\
\text { Dor leve e moderada } \\
\text { Dor visceral }\end{array}$ & $\begin{array}{l}8 \\
7 \\
4 \\
2\end{array}$ & $\begin{array}{l}38,1 \\
33,4 \\
19,0 \\
9,5\end{array}$ & $\begin{array}{l}18 \\
7 \\
- \\
-\end{array}$ & $\begin{array}{l}72 \\
28 \\
- \\
-\end{array}$ & \\
\hline Conhecimento de escalas de & $\operatorname{Sim}$ & 12 & 36,4 & 25 & 69,4 & \\
\hline dor & Não & 21 & 63,6 & 11 & 30,6 & 0,012 \\
\hline $\begin{array}{l}\text { Utilização de escalas de dor du- } \\
\text { rante o cuidado }\end{array}$ & $\underset{\text { Sim }}{\text { Sĩo }}$ & $\frac{6}{6}$ & $\begin{array}{l}50,0 \\
50,0\end{array}$ & $\frac{20}{5}$ & $\begin{array}{l}86,9 \\
13,1\end{array}$ & 0,137 \\
\hline
\end{tabular}

* número de casos válidos

${ }^{* *}$ dentre os que responderam sim 
Quanto ao conhecimento dos profissionais de enfermagem em relação aos fatores que agravam a dor, em ambos os questionários, evidencia que não existe diferença entre as respostas do Q1 e Q2 $(\mathrm{p}=0,1906)$.Os principais fatores que agravam a dor em relação a sentimentos e emoções agravam a dor foram: o estado emocional / psicológico abalado (Q1=55,5\%; Q2=50,0\%) e medo (Q1=27,8\%; $\mathrm{Q} 2=11,5 \%)$.

Quanto à diminuição da função e atividade do corpo como fator de agravo a dor, houve uma inversão de respostas, demonstrando que a dependência entre o tipo de questionário $(\mathrm{p}=0,0009)$. Em Q1 $72,2 \%$ responderam a não mudança de decúbito, no Q2 71, 4\% assinaram a evolução/fatores da doença.
Já em relação ao manejo com a medicação, demonstra que existe dependência entre o tipo de questionário $(\mathrm{p}=0,0235) .19(86,4 \%)$ atribuíram ao atraso no horário da medicação como fator que agrava a dor. No Q2, dos 22 respondentes, $11(50,0 \%)$ atribuíram ao atraso no horário da medicação, 11 (37,5\%) disseram que é por ter prescrita somente medicação se necessário agrava a dor.

Quanto ao indicador atitude e preparo do profissional, em ambos os questionários evidenciou que não existe diferença entre as respostas do Q1 e Q2 ( $\mathrm{p}=0,2544)$. Os principais fatores citados foram: Falta de capacitação $(\mathrm{Q} 1=53,8 \%$; Q2= $29,4 \%$ ), falta de sensibilidade (Q1=30,8\%; Q2= $29,4 \%)$.

Tabela 3 - Conhecimento dos profissionais de enfermagem dos fatores que agravam a dor, em um hospital oncológico, Cuiabá-MT, 2012

\begin{tabular}{|c|c|c|c|c|c|c|}
\hline Variáveis & & \multicolumn{2}{|c|}{ Q1 } & \multicolumn{2}{|c|}{ Q2 } & p \\
\hline \multirow{5}{*}{$\begin{array}{l}\text { Sentimentos e } \\
\text { emoções }\end{array}$} & & $\mathrm{n}^{*}$ & $\%$ & $\mathrm{n}^{*}$ & $\%$ & \multirow{5}{*}{0,1906} \\
\hline & Estado emocional abalado & 10 & 55,5 & 13 & 50,0 & \\
\hline & Medo & 5 & 27,8 & 3 & 11,5 & \\
\hline & Ansiedade & 3 & 16,7 & 10 & 38,5 & \\
\hline & Total & 18 & 100,0 & 26 & 100,0 & \\
\hline \multirow{3}{*}{$\begin{array}{l}\text { Função e ativida- } \\
\text { de do corpo }\end{array}$} & Não mudança de decúbito & 13 & 72,2 & 4 & 14,3 & \\
\hline & Evolução/ Fatores da doença & 5 & 27,8 & 20 & 71,4 & \multirow{2}{*}{0,0009} \\
\hline & Total & 18 & 100,0 & 24 & 100,0 & \\
\hline \multirow{3}{*}{$\begin{array}{l}\text { Manejo da me- } \\
\text { dição }\end{array}$} & Atraso no horário da medicação & 19 & 86,4 & 11 & 50,0 & \multirow{3}{*}{0,0235} \\
\hline & Medicação se necessário & 3 & 13,6 & 11 & 50,0 & \\
\hline & Total & 22 & 100,0 & 22 & 100,0 & \\
\hline $\begin{array}{c}\text { Atitude e Preparo } \\
\text { do profissional }\end{array}$ & $\begin{array}{c}\text { Falta de capacitação } \\
\text { Falta de sensibilidade } \\
\text { Assistência não humanizada } \\
\text { Total }\end{array}$ & $\begin{array}{c}7 \\
4 \\
2 \\
13 \\
\end{array}$ & $\begin{array}{l}53,8 \\
30,8 \\
15,4 \\
100.0 \\
\end{array}$ & $\begin{array}{c}5 \\
5 \\
7 \\
17 \\
\end{array}$ & $\begin{array}{r}29,4 \\
29,4 \\
41,1 \\
100.0 \\
\end{array}$ & 0,2544 \\
\hline
\end{tabular}

* número de casos válidos

Quanto ao conhecimento dos profissionais de enfermagem sobre as medidas que aliviam a dor, em ambos os questionários, a realização de medicação conforme prescrição medica apresentou maiores percentuais $(\mathrm{Q} 1=55,5 \%$; $\mathrm{Q} 2=58,3$ ). Para as medi- das não farmacológicas, no Q1 o apoio psicológico (30,5\%) e mudança de decúbito sobressaíram, enquanto no Q2 o apoio psicológico (27,7\%), seguido de massagem terapêutica $(13,8 \%)$ e mudança de decúbito $(13,3 \%)$, tiveram maiores proporções.

Tabela 4 - Conhecimento dos profissionais de enfermagem sobre medidas farmacológicas e não farmacológicas no alívio da dor no paciente oncológico, Cuiabá-MT, 2012

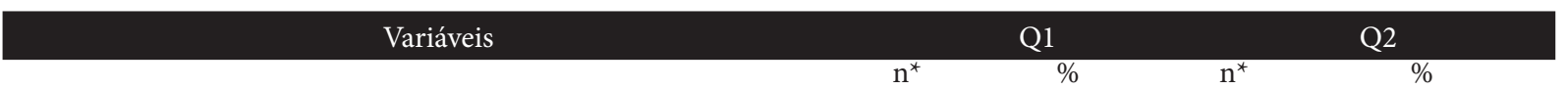

Medidas farmacológicas 
Realizar medicação conforme prescrição médica

20

55,5

21

58,3

Medidas não farmacológicas

Mudança de decúbito

Exercício de relaxamento

Toque terapêutico

Massagem terapêutica

Comunicação terapêutica

Apoio psicológico
Oração

* Mais de uma alternativa.

Na tabela 5 o grau de concordância para as questões: você faz uso de medidas não farmacológicas, conhece as escalas de dor existentes e a di- minuição da função e atividade do corpo agrava a dor, apresentaram maior concordância em relação às demais.

Tabela 5 - Grau de concordância das questões relacionadas ao conhecimento dos profissionais de enfermagem quanto a dor no paciente oncológico, Cuiabá-MT, 2012

\begin{tabular}{|c|c|c|c|c|c|}
\hline Variáveis & $\mathbf{n}^{*}$ & positivas & negativas & $\begin{array}{l}\text { Proporção de } \\
\text { concordância }\end{array}$ & Kappa \\
\hline $\begin{array}{l}\text { Conhece as escalas de dor existentes? } \\
\text { A diminuição da função e atividade do }\end{array}$ & 33 & 36,4 & 36,4 & 0,728 & 0,492 \\
\hline corpo agrava a dor? & 34 & 76,5 & 8,8 & 0,853 & 0,465 \\
\hline $\begin{array}{l}\text { O cuidado com o horário da medicação } \\
\text { interfere na dor? }\end{array}$ & 34 & 76,5 & 2,9 & 0,794 & 0,119 \\
\hline $\begin{array}{c}\text { A atitude e preparo do profissional } \\
\text { interfere na dorr? }\end{array}$ & 34 & 67,6 & 8,8 & 0,764 & 0,284 \\
\hline $\begin{array}{c}\text { Você faz uso de medidas não } \\
\text { farmacológicas? }\end{array}$ & 35 & 22,9 & 54,3 & 0,772 & 0,495 \\
\hline
\end{tabular}

\section{DISCUSSÃO}

Entre a população do estudo $88,9 \%$ dos profissionais eram do sexo feminino, mostrando que as mulheres ainda constituem a maioria na equipe de enfermagem ${ }^{2}$. Quanto ao tempo de experiência cuidando de pacientes com câncer e a realização de curso, nesse estudo foi mostrado que a maioria tem menos de 2 anos e a grande proporção $(77,8 \%)$, nunca havia realizado cursos de atualização na área. Dessa forma o pouco tempo de experiência profissional, reforça a relevância do estudo, pois a identificação do nível de conhecimento destes profissionais torna-se um parâmetro para o planejamento da assistência de enfermagem no manejo da dor no paciente oncológico, e o pouco tempo de experiência cuidando de pacientes com câncer, pode interferir diretamente na efetividade das ações de enfermagem em relação ao manejo da dor ${ }^{5-7}$.

Em relação ao conhecimento dos tipos de dor evidenciado em ambos os questionários foi en- contrado que uma boa proporção os profissionais de enfermagem que relataram que tem esse conhecimento, evidenciou que não existe diferença entre as resposta de ambos os questionários. No entanto, foi diferente do estudo realizado em um hospital de ensino nos Estados Unidos, onde 62\% dos profissionais de saúde (enfermeiros, farmacêuticos e médicos) relataram não possuir conhecimentos suficientes sobre dor e analgesia ${ }^{8}$.

Apesar do alto percentual de respostas positivas neste estudo quanto ao conhecimento da dor, e o fato de o alívio desta ser uma parte essencial do cuidado dos profissionais de enfermagem, a literatura tem apontado que os profissionais não têm conseguido aliviá-la adequadamente por não reconhecê-la, por não conseguir tratá-la e por aceitar que a dor severa faz parte da doença e do tratamento. Estes empecilhos para o tratamento adequado da dor são ocasionados pela falta de conhecimento sobre a sua avaliação e manejo ${ }^{9,10}$. 
Os achados quanto ao conhecimento dos profissionais sobre as escalas de dor, evidenciaram uma inversão nas respostas, demonstrando que a dependência entre o tipo de questionário. Este resultado pode ser atribuído à falta de informação dos profissionais de enfermagem sobre a utilização da escala da dor, em decorrência da inexistência da temática nos projetos curriculares dos cursos de formação em enfermagem, ocasionando lacunas na formação acadêmica. Outro aspecto também se refere ao fato de que a avaliação da dor de forma sistemática não tem sido uma rotina nos serviços, levando os profissionais a subestimarem a frequência da ocorrência de dor, bem como ignorarem o impacto devastador desta para o indivíduo ${ }^{9,11-12}$

Um estudo realizado em Santa Catarina, em 2008, que teve como objetivo avaliar a percepção e conhecimento de farmacêuticos, médicos e enfermeiros em relação ao tema dor, assim como a preocupação com a mesma e com o conforto do paciente, evidenciou que, embora demonstrando maior conhecimento das escalas em relação aos demais profissionais, metade dos enfermeiros pesquisados não citou ou desconheciam as escalas para mensurar a dor ${ }^{13}$.

$\mathrm{Na}$ análise das respostas com base na experiência prática dos respondentes, todos citaram fatores que agravam a dor. Isso demonstra que nem todos os fatores eram de total desconhecimento dos participantes, mesmo que esses não tenham sido lembrados no levantamento dos conhecimentos prévios no questionário aberto. Mas reconhecer e admitir a possibilidade desses fatores no momento em que se deparou com as opções de resposta do questionário fechado pode ser entendido como uma forma, mesmo que empírica, de conhecimento de tais fatores. Assim, dentre eles o mais citado tanto em Q1 e Q2 foi estado emocional/ psicológico abalado, em relação a sentimentos e emoções que agravam a dor. Não houve diferença entre as respostas do Q1 e Q2. Este reconhecimento do emocional interferindo no físico é muito importante na assistência e na relação doente/equipe de enfermagem, já que a enfermagem não vê mais um órgão doente apenas, e sim um paciente abalado emocionalmente, desencadeando emoções que fragilizam, causam dor, angústia e medo, pois para o cliente a confirmação de que se tem um câncer é sempre associada à morte ${ }^{14}$.
Em relação à diminuição da função e atividade do corpo que agrava da dor, os principais fatores apontados nos questionários foram: não mudança decúbito e evolução da patologia. Contudo houve uma divergência em relação à resposta "mudança de decúbito" entre os questionários, pois no segundo é o menos citado como fator de agravo a dor. Dessa forma foi mostrado que existe a dependência entre o tipo de questionário, o que indica que os profissionais valorizam muito mais a mudança de decúbito como ação efetiva para a prevenção de úlceras de pressão, e outra possibilidade se deve por terem encontrado no questionário fechado várias opções de resposta, possibilitando relembrar aspectos que não haviam pensado anteriormente ${ }^{15}$.

Já em relação ao manejo com a medicação, houve uma divergência dos questionários, pois o atraso da medicação foi a primeira opção dos profissionais de enfermagem em Q1, já em Q2 metade dos respondentes relacionaram ao atraso na medicação e prescrição medica se necessário. Assim, existe a dependência entre o tipo de questionário ( $\mathrm{p}=0,0235)$, o que indica que a medicação está prescrita pelo medico, tanto de horário como se necessário, envolverá tempo para profissional de enfermagem providenciar o analgésico prescrito e prepará-lo para a administração . Este fato exige um período de espera do paciente pelo medicamento, o que resulta no aumento da intensidade da dor e sofrimento do paciente ${ }^{(14)}$.

Quando se trata do conhecimento dos profissionais de enfermagem de medidas que aliviam a dor, administrar medicação é o de maior representatividade como alívio de dor na amostra apresentada. Dessa forma, fica evidente que a equipe enfermagem reconhece como prioridade a terapia farmacológica, no entanto, também citam mais timidamente algumas terapias não farmacológicas. O estudo de Ying Xue et al. ${ }^{8}$ mostrou que 69\% enfermeiros acreditava que a maioria da dor dos pacientes $(69 \%)$ poderia ser aliviados com o tratamento adequado com medicamentos.

Dessa forma para esses profissionais a "medicação" parece à melhor forma para controlar e combater a dor. No entanto, existem outras medidas que podem ser usadas pela enfermagem, que independem da intervenção médica. Nem todas as técnicas não farmacológicas substituem os 
analgésicos, porém, em doentes que necessitam de tratamento farmacológico, estas podem ser úteis na redução da dosagem da medicação e na diminuição da dor enquanto a medicação não produz o efeito terapêutico ${ }^{16}$.

Os dados obtidos demonstram a valorização do tratamento farmacológico em detrimento do tratamento não farmacológico no manejo da dor entre grande parte dos profissionais da enfermagem. Esse achado vai ao encontro dos resultados com um estudo realizado com equipe de enfermagem da UTI, de um Hospital Universitário de grande porte da cidade de Curitiba, que objetivou identificar como a equipe de enfermagem avalia a dor, e qual intervenção é realizada para seu alívio. Dentre as intervenções, a mais citada foi utilizada é a medicamentosa conforme prescrição médica com $41,17 \%$, e percentagens menores para outras ações como avisar o médico responsável pelo paciente quando a medicação prescrita não foi suficiente $(34,13 \%)$; proporcionar conforto $(24,53 \%)$ e fazer bolsa de água quente $(1,17 \%)$, pois a bolsa de água quente somente deve ser utilizada em algumas situações de dor, e não em qualquer queixa de dor ${ }^{4}$.

A realização do grau de concordância entre os questionários, evidenciou a falta de conhecimento no manejo da dor em ambos os questionários, onde tanto as respostas subjetivas como as objetivas mostraram baixo conhecimento no manejo e tratamento da dor. Esses achados reforçam o que a prática parece demonstrar, ou seja, os profissionais não estão preparados para atuarem no manejo da dor do paciente oncológico, assim, realizam a assistência utilizando-se de um conhecimento empírico e não do conhecimento fundamentado em princípios científicos e fundamental para a qualidade da assistência.

$\mathrm{O}$ inadequado manejo da dor pela equipe de enfermagem é influenciado por diferentes aspectos como, pelo déficit de conhecimento, pela comunicação inadequada entre equipe de enfermagem e paciente/família, pelas dificuldades de transpor barreiras para um melhor manejo da dor, ou seja, pela falta de valorização de medidas não farmacológicas, sobrecarga de trabalho, experiência profissional e pessoal sobre a dor, bem como a habilidade dos pacientes oncológicos para expressar a dor. Estes fatores nos alertam para a com- plexidade de elementos que interferem no manejo da dor ao paciente com câncer, contribuindo para reflexões da equipe de enfermagem ${ }^{17}$.

\section{CONCLUSÃO}

O estudo evidenciou a falta de conhecimento no manejo da dor em ambos os questionários. Assim, indica que a equipe de enfermagem deve ser treinada no que se refere ao manejo da dor. A conscientização e incentivo para a formação na prática profissional dos recém-introduzidos no mercado de trabalho, bem como dos que já exercem suas atividades profissionais, necessitam ser estimulada por meio de educação permanente visando ampliar o conhecimento nesta área. No entanto, isso demanda tempo e investimento para que se possa observar a mudança na prática, mas instituir a dor como o quinto sinal vital se faz necessário, para que o manejo da dor faça parte da rotina da enfermagem.

Dessa forma, destaca-se à introdução e discussão de conteúdos relacionados à temática da dor em pacientes com câncer, refere-se à realização de atividades de extensão nos serviços, discutindo conteúdos e métodos não-farmacológicos para ao alívio da dor. Vale ressaltar ainda que a condução de pesquisas nesta área, com a coleta de dados junto aos profissionais de enfermagem, pode contribuir para suscitar discussões e reflexões acerca do tema pela equipe como um todo.

Considerando a dor um evento que gera impacto na qualidade de vida do paciente com câncer, pode-se dizer que o profissional de enfermagem não tem conhecimento suficiente no manejo da dor do paciente oncológico, com fragilidades para avaliar a dor por meio de escalas. Somente por meio de capacitação e conscientização desses profissionais podemos mudar esse cenário, desmistificando que a dor só pode ser aliviada por analgésicos de uso contínuo, e mostrando que a utilização de medidas não farmacológicas pode contribuir para controlar e aliviar a dor.

\section{REFERENCIAS}

1. Morete MC, Minson FP. Instrumentos para a avaliação da dor em pacientes oncológicos. Rev dor. 2010; 11(1): 74-80. 
2. Alves VS, Santos TS, Trezza MCSF, Santos RM, Monteiro FS. Conhecimento de Profissionais da Enfermagem sobre Fatores que Agravam e Aliviam a Dor Oncológica. Revista Brasileira de Cancerologia 2011; 57(2):199-206.

3. Rheingans JI. A systematic review of nonpharnmacologic adjunctive therapies for symptom management in children with câncer. J Ped Oncol Nurs 2007; 24 (2): 81-94.

4. Magalhães PAP, Mota FA, Saleh CMR, Dal Secco LM, Fusco SRG, Gouvêa AL. Percepção dos profissionais de enfermagem frente à identificação, quantificação e tratamento da dor em pacientes de uma unidade de terapia intensiva de trauma. Rev. dor. 2011; 12(3): 221-225.

5. Fellowes D, Barnes K, Wilkinson S. Aromaterapia y masajes para el alivio sintomático de los pacientes oncológicos (Cochrane Review). In: La Biblioteca Cochrane Plus, Issue 3, 2008.

6. Abreu MAV, Reis PED, Gomes IP, Rocha PRS. Manejo não farmacológico da dor em pacientes com câncer: revisão sistemática / Non pharmacologic pain management on oncologic patients: systematic review. Online braz. j. nurs. (Online); 8(1)2009.

7. Pedroso RA, Celich KLS. Dor: quinto sinal vital, um desafio para o cuidar em enfermagem. Texto contexto - enferm. 2006; 15(2): 270-276.

8. Xue Y1, Schulman-Green D, Czaplinski C, Harris D, McCorkle R. Pain Attitudes and Knowledge Among RNs, Pharmacists, and Physicians on an Inpatient Oncology Service. Clinical Journal of Oncology Nursing; 11(5): 687-95, 2007.

9. Alves VS, Santos TS, Trezza MCSF, Santos RM, Monteiro FS. Conhecimento de profissionais da Enfermagem sobre fatores que agravam e aliviam a Dor Oncológica. Rev. bras. cancerol; 57(2): 199-206, abr./jun. 2011.

10. Chotolli MR, Luize PB. Métodos não farmacológicos no controle da dor oncológica pediátrica: visão da equipe de enfermagem. Rev. dor. 2015; 16(2): 109-113.

11. Waterkemper R, Reibnitz KS. Cuidados paliativos: a avaliação da dor na percepção de enfermeiras. Rev Gaucha Enferm Porto Alegre. 2010; 31(1):84-91.

12. Francischinelli AGB, Modena T, Morete MC. Conhecimento dos profissionais de enfermagem quanto às medidas não farmacológicas para o alívio da dor nos pacientes pediátricos. Revista Dor. 2009; 10(1):19-24.

13. Kulkamp IC, Barbosa CG, Bianchini KC. Percepção de profissionais da saúde sobre aspectos relacionados à dor e utilização de opióides: um estudo qualitativo. Ciênc Saúde Colet; 2008, 13 (1): 721-31.
14. Ribeiro MCO, Pereira CU, Sallum AMC, Alves JAB, Albuquerque MF, Fujishima PA. Conhecimento de médicos e enfermeiros sobre dor em pacientes submetidos à craniotomia. Rev. Latino-Am. Enfermagem. 2012; 20(6): 1057-1063.

15. Nascimento LA, Santos MR, Aroni P, Martins MB, Kreling MCGD. Manejo da dor e dificuldades relatadas pela equipe de enfermagem na administração de opióides. Rev. Eletr. Enf. 2011;13(4):714-20.

16. Oliveira A L, Palma SN, CBAS. Manuseio da dor crônica em pacientes oncológicos pela equipe de enfermagem. Rev. dor. 2016; 17(3): 219-222.

17. Cunha FF, Rêgo LP. Enfermagem diante da dor oncológica. Rev. dor. 2015; 16(2): 142-145.

\section{Como citar este artigo:}

Lara HCAA, Rubira EA, Marcon SR, Oliveira JRT, Britto AS, Alexandre RMS. Conhecimento dos profissionais de enfermagem no manejo da dor de pacientes oncológicos. Rev. Aten. Saúde. 2018;16(58):49-56. 